Bull. Chem. Soc. Ethiop. 2019, 33(1), 149-158.

ISSN 1011-3924

(c) 2019 Chemical Society of Ethiopia and The Authors

Printed in Ethiopia

DOI: https://dx.doi.org/10.4314/bcse.v33i1.15

\title{
CAFFEINE AS A NATURALLY GREEN AND BIODEGRADABLE CATALYST PROMOTED CONVENIENT AND EXPEDIENT SYNTHETIC ROUTE FOR THE SYNTHESIS OF POLYSUBSTITUTED DIHYDRO-2-OXYPYRROLES
}

\author{
Farzaneh Mohamadpour*
}

Young Researchers and Elite Club, Shiraz Branch, Islamic Azad University, Shiraz, Iran

(Received July 31, 2018; Revised January 10, 2019; Accepted January 16, 2019)

\begin{abstract}
A green, convenient, high yielding and one-pot procedure for synthesis of high substituted dihydro-2-oxypyrroles by domino four-component condensation reaction between aromatic/aliphatic amines, dialkyl acetylenedicarboxylate and formaldehyde in the presence of a catalytic amount of caffeine as a green, natural, expedient and biodegradable catalyst under ambient temperature was studied. The salient features of this green approach are simplicity of operation and work-up procedures with no necessity of chromatographic purification steps, use of safe, non-volatile, non-corrosive and green catalyst, the availability and easy to handle of this solid catalyst, one-pot reaction, economical and clean synthesis.
\end{abstract}

KEY WORDS: Caffeine, Green catalyst, Polysubstituted dihydro-2-oxypyrroles, Ambient temperature, Simple work-up

\section{INTRODUCTION}

Polyfunctionalized heterocyclic compounds are playing important roles in drug discovery processes and in the analysis of drugs. In particular, pyrroles [1,2] and their analogues have been receiving attention owing to their biological and pharmacological properties [3] such as human cytomegalovirus (HCMV) protease [4], human cytosolic carbonic anhydrase isozymes [5], they have been used as PI-091 [6], and cardiac cAMPphosphodiestrase [7], many of the alkaloids have pyrrole rings [8]. Also, these rings have been utilized as Oteromycin [9]. They exhibit various biological activities, for example (imidazolylphenyl) pyrrol-2-one [7] and VEGF-R, a vascular endothelial growth factor receptor [10]. Some of them with biological properties have been shown in Figure 1.

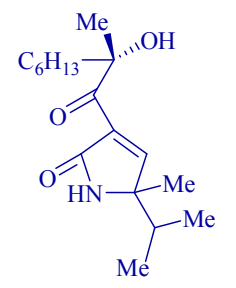

PI-091

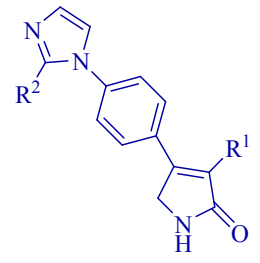

$\mathbf{R}^{1}=\mathbf{M e}, \mathbf{E t}$

$\mathbf{R}^{2}=\mathbf{H}, \mathbf{M e}$

(imidazolylphenyl) pyrrol-2-one

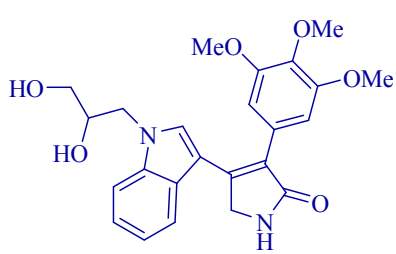

VEGF-R

Figure 1. Biologically active compounds with dihydro-2-oxypyrrole rings.

To date, methods for the synthesis of high substituted dihydro-2-oxypyrroles have been reported using MCRs in the presence of various catalysts such as $\mathrm{I}_{2}$ [11], $\mathrm{InCl}_{3}$ [12], [n$\left.\mathrm{Bu}_{4} \mathrm{~N}\right]\left[\mathrm{HSO}_{4}\right.$ ] [13], $\mathrm{Al}\left(\mathrm{H}_{2} \mathrm{PO}_{4}\right)_{3}$ [14], $\mathrm{AcOH}$ [15], $\mathrm{Cu}(\mathrm{OAc})_{2} \cdot \mathrm{H}_{2} \mathrm{O}$ [16], oxalic acid dihydrate [17], $\mathrm{ZrCl}_{4}$ [18] and $\mathrm{Fe}_{3} \mathrm{O}_{4} @$ nano-cellulose- $\mathrm{OPO}_{3} \mathrm{H}$ [19]. Some of these methods have

*Corresponding author. E-mail: mohamadpour.f.7@gmail.com

This work is licensed under the Creative Commons Attribution 4.0 International License 
limitations such as long time reactions, low yields, the use of strongly acidic conditions, high temperature, difficulty work-up, toxic and expensive catalysts.

The detection and measurement of caffeine (CAF) (Figure 2) or trimethylxanthine alkaloid, as a central nervous system and metabolic stimulant [20], have attracted the attention of many researchers. This compound is chemically related to the adenine and guanine bases of deoxyribonucleic acid (DNA) and ribonucleic acid (RNA). In the case of multifunctional molecules, such as caffeine, there may be several sites for protonation (Figure 3). Proton affinity (PA) is one of the most important thermodynamic quantities that link the thermochemistry of ions to that of the neutral molecules [21].

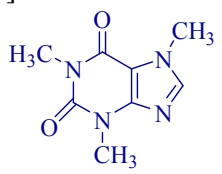

Figure 2. Structure of Caffeine.<smiles>Cn1c(=O)c2c(ncn2C)n(C)c1=O</smiles><smiles>C=[In]C</smiles><smiles>Cn1c(=O)c2c(nc3n(C)c(I)cn23)n(C)c1=O</smiles>

Figure 3. Protonation of caffeine.

The design of multi-component reactions (MCRs) [22-32] has received great attention from research groups in medicinal chemistry, drug discovery and materials science due to their significant advantages over conventional linear-type synthesis, including simple procedures, environmental friendliness, atom economy, and the ability to generate architecturally complex molecules in one synthetic step.

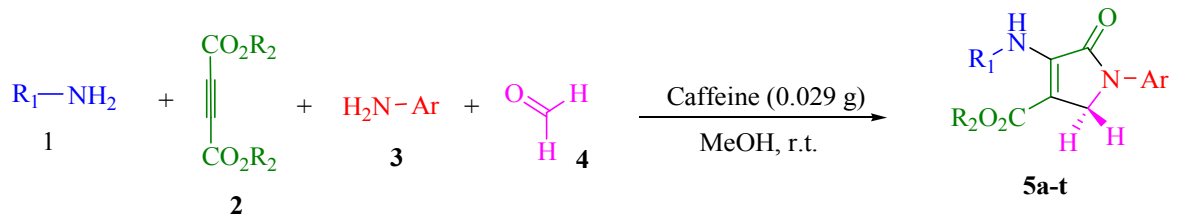

Scheme 1. Synthesis of polysubstituted dihydro-2-oxypyrroles.

Finally, due to the above considerations and in continuation of our work on the development of useful and green synthetic methodology for the preparation of biologically active heterocyclic compounds using of caffeine as catalyst [33], we report herein, synthesis of polysubstituted dihydro-2-oxypyrroles via one-pot, four condensation domino reaction between aromatic/ aliphatic amines (1 and 3), dialkyl acetylenedicarboxylate $\mathbf{2}$ and formaldehyde $\mathbf{4}$ in the presence 
of caffeine as a cost effective, green, biodegradable and readily catalyst under mild conditions (Scheme 1).

\section{EXPERIMENTAL}

Melting points all compounds were determined using an Electro thermal 9100 apparatus. Also, nuclear magnetic resonance, ${ }^{1} \mathrm{H}$ NMR spectra were recorded on a Bruker DRX-400 Avance instrument with $\mathrm{CDCl}_{3}$ as solvent. All reagents and solvents were purchased from Merck, Fluka and Acros chemical companies were used without further purification.

General procedure for preparation of substituted dihydro-2-oxypyrroles (5a-t)

A mixture of amine $1(1.0 \mathrm{mmol})$ and dialkyl acetylenedicarboxylate $2(1.0 \mathrm{mmol})$ was stirred in $\mathrm{MeOH}(3 \mathrm{~mL})$ for $15 \mathrm{~min}$. Next, amine $3(1.0 \mathrm{mmol})$ and formaldehyde $4(1.5 \mathrm{mmol})$ and caffeine $(15 \mathrm{~mol} \%, 0.029 \mathrm{~g})$ were added and the reaction was stirred for appropriate time. After completion of the reaction (by thin layer chromatography TLC), the mixture was separated by filtration and the solid washed with ethanol $(3 \times 2 \mathrm{~mL})$ with no column chromatographic separation to give pure compounds (5a-t). The products were characterized by comparison of spectroscopic data $\left({ }^{1} \mathrm{HNMR}\right)$. Spectra data some of known products are represented below.

Methyl4-(4-fluoroyphenylamino)-1-(4-fluorophenyl)-2,5-dihydro-5-oxo-1H-pyrrole-3-carboxylate (5c). Yield: $87 \%$; m.p. $164-165{ }^{\circ} \mathrm{C} ;{ }^{1} \mathrm{H}$ NMR (400 MHz, $\left.\mathrm{CDCl}_{3}\right): 3.79\left(3 \mathrm{H}, \mathrm{s}, \mathrm{OCH}_{3}\right), 4.52$ $\left(2 \mathrm{H}, \mathrm{s}, \mathrm{CH}_{2}-\mathrm{N}\right), 7.04(2 \mathrm{H}, \mathrm{t}, J=8.4 \mathrm{~Hz}, \mathrm{ArH}), 7.08-7.16(4 \mathrm{H}, \mathrm{m}, \mathrm{ArH}), 7.73-7.76(2 \mathrm{H}, \mathrm{m}, \mathrm{ArH})$, $8.05(1 \mathrm{H}, \mathrm{s}, \mathrm{NH})$.

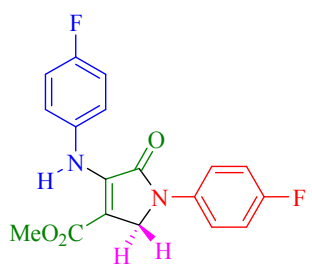

Methyl4-(4-methoxyphenylamino)-1-(4-methoxyphenyl)-2,5-dihydro-5-oxo-1H-pyrrole-3-carboxylate (5g). Yield: 85\%; m.p. 175-177 ${ }^{\circ} \mathrm{C} ;{ }^{1} \mathrm{H}$ NMR (400 MHz, $\left.\mathrm{CDCl}_{3}\right): 3.77\left(3 \mathrm{H}, \mathrm{s}, \mathrm{CH}_{3}\right)$, $3.83\left(6 \mathrm{H}, \mathrm{s}, 2 \mathrm{OCH}_{3}\right), 4.50\left(2 \mathrm{H}, \mathrm{s}, \mathrm{CH}_{2}-\mathrm{N}\right), 6.89(4 \mathrm{H}, \mathrm{d}, J=17.6 \mathrm{~Hz}, \operatorname{ArH}), 7.13(1 \mathrm{H}, \mathrm{s}, \operatorname{ArH})$ ,7.68 $(1 \mathrm{H}, \mathrm{s}, \mathrm{ArH}), 8.03(1 \mathrm{H}, \mathrm{s}, \mathrm{NH})$.

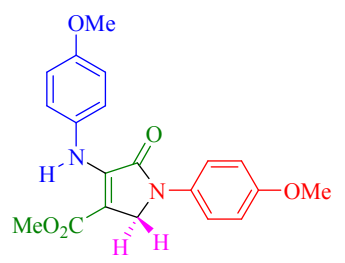

Ethyl4-(4-methoxyphenylamino)-1-(4-methoxyphenyl)-2,5-dihydro-5-oxo-1H-pyrrole-3-carboxylate (5h). Yield: 87\%; m.p. $150-152{ }^{\circ} \mathrm{C} ;{ }^{1} \mathrm{H}$ NMR $\left(400 \mathrm{MHz}, \mathrm{CDCl}_{3}\right): 1.26(3 \mathrm{H}, \mathrm{t}, J=7.2 \mathrm{~Hz}$, $\left.\mathrm{CH}_{2} \underline{\mathrm{CH}}_{3}\right), 3.83\left(6 \mathrm{H}, \mathrm{s}, 2 \mathrm{OCH}_{3}\right), 4.23\left(2 \mathrm{H}, \mathrm{q}, J=7.2 \mathrm{~Hz}, \underline{\mathrm{CH}}_{2} \mathrm{CH}_{3}\right), 4.50\left(2 \mathrm{H}, \mathrm{s}, \mathrm{C}_{2}-\mathrm{N}\right), 6.87$ $(2 \mathrm{H}, \mathrm{d}, J=8.8 \mathrm{~Hz}, \mathrm{ArH}), 6.93(2 \mathrm{H}, \mathrm{d}, J=8.8 \mathrm{~Hz}, \mathrm{ArH}), 7.12(2 \mathrm{H}, \mathrm{d}, J=8.8 \mathrm{~Hz}, \mathrm{ArH}), 7.69$ $(2 \mathrm{H}, \mathrm{d}, J=8.8 \mathrm{~Hz}, \mathrm{ArH}), 8.02(1 \mathrm{H}, \mathrm{s}, \mathrm{NH})$. 


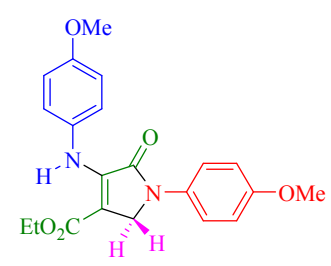

Methyl4-(4-methylphenylamino)-1-(4-methylphenyl)-2,5-dihydro-5-oxo-1H-pyrrole-3-carboxylate (5k). Yield: 89\%; m.p. 176-178 ${ }^{\circ} \mathrm{C}$; ${ }^{1} \mathrm{H}$ NMR (400 MHz, $\left.\mathrm{CDCl}_{3}\right): 2.36\left(6 \mathrm{H}, \mathrm{s}, 2 \mathrm{CH}_{3}\right), 3.77$ $\left(3 \mathrm{H}, \mathrm{s}, \mathrm{OCH}_{3}\right), 4.52\left(2 \mathrm{H}, \mathrm{s}, \mathrm{CH}_{2}-\mathrm{N}\right), 7.06(2 \mathrm{H}, \mathrm{d}, J=8.4 \mathrm{~Hz}, \mathrm{ArH}), 7.14(2 \mathrm{H}, \mathrm{d}, J=8.4 \mathrm{~Hz}$, $\mathrm{ArH}), 7.21(2 \mathrm{H}, \mathrm{d}, J=8.4 \mathrm{~Hz}, \mathrm{ArH}), 7.68(2 \mathrm{H}, \mathrm{d}, J=8.8 \mathrm{~Hz}, \mathrm{ArH}), 8.03(1 \mathrm{H}, \mathrm{s}, \mathrm{NH})$.

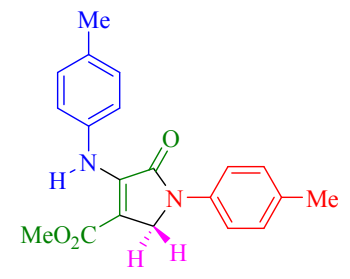

Ethyl4-(4-methylphenylamino)-1-(4-methylphenyl)-2,5-dihydro-5-oxo-1H-pyrrole-3-carboxylate (5l): Yield: $86 \%$; m.p. $132-134{ }^{\circ} \mathrm{C}$; ${ }^{1} \mathrm{H}$ NMR $\left(400 \mathrm{MHz}, \mathrm{CDCl}_{3}\right): 1.25(3 \mathrm{H}, \mathrm{t}, J=7.2 \mathrm{~Hz}$, $\left.\mathrm{CH}_{2} \underline{\mathrm{CH}}_{3}\right), 2.37\left(6 \mathrm{H}, \mathrm{s}, 2 \mathrm{CH}_{3}\right), 4.23\left(2 \mathrm{H}, \mathrm{q}, J=7.2 \mathrm{~Hz}, 2 \mathrm{CH}_{2} \mathrm{CH}_{3}\right), 4.53\left(2 \mathrm{H}, \mathrm{s}, \mathrm{C}_{2}-\mathrm{N}\right), 7.06$ $(2 \mathrm{H}, \mathrm{d}, J=8.4 \mathrm{~Hz}, \mathrm{ArH}), 7.14(2 \mathrm{H}, \mathrm{d}, J=8.4 \mathrm{~Hz}, \mathrm{ArH}), 7.21(2 \mathrm{H}, \mathrm{d}, J=8.4 \mathrm{~Hz}, \mathrm{ArH}), 7.69$ $(2 \mathrm{H}, \mathrm{d}, J=8.4 \mathrm{~Hz}, \mathrm{ArH}), 8.01(1 \mathrm{H}, \mathrm{s}, \mathrm{NH})$.

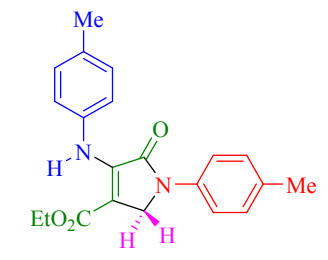

Methyl4-(4-ethylphenylamino)-1-(4-ethylphenyl)-2,5-dihydro-5-oxo-1H-pyrrole-3-carboxylate (5m). Yield: $82 \%$; m.p. 126-128 ${ }^{\circ} \mathrm{C} ;{ }^{1} \mathrm{H}$ NMR $\left(400 \mathrm{MHz}, \mathrm{CDCl}_{3}\right): 1.26(6 \mathrm{H}, \mathrm{t}, J=2.4 \mathrm{~Hz}$, $\left.2 \mathrm{CH}_{2} \mathrm{CH}_{3}\right), 2.67\left(4 \mathrm{H}, \mathrm{q}, J=7.2 \mathrm{~Hz}, 2 \mathrm{CH}_{2} \mathrm{CH}_{3}\right), 3.76\left(3 \mathrm{H}, \mathrm{s}, 2 \mathrm{OCH}_{3}\right), 4.53\left(2 \mathrm{H}, \mathrm{s}, \mathrm{CH}_{2}-\mathrm{N}\right), 7.09$ $(2 \mathrm{H}, \mathrm{d}, J=8.4 \mathrm{~Hz}, \mathrm{ArH}), 7.17(2 \mathrm{H}, \mathrm{d}, J=8.4 \mathrm{~Hz}, \mathrm{ArH}), 7.24(2 \mathrm{H}, \mathrm{d}, J=8.8 \mathrm{~Hz}, \mathrm{ArH}), 7.70$ $(2 \mathrm{H}, \mathrm{d}, J=8.8 \mathrm{~Hz}, \mathrm{ArH}), 8.05(1 \mathrm{H}, \mathrm{s}, \mathrm{NH})$.

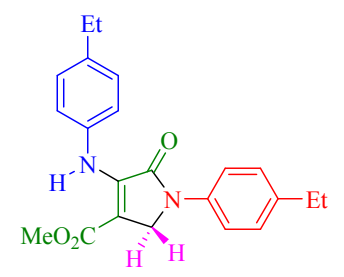

Ethyl4-(4-ethylphenylamino)-1-(4-ethylphenyl)-2,5-dihydro-5-oxo-1H-pyrrole-3carboxylate

(5n). Yield: 84\%; m.p. 102-104 ${ }^{\circ} \mathrm{C} ;{ }^{1} \mathrm{H}$ NMR (400 MHz, $\left.\mathrm{CDCl}_{3}\right): 1.24$ (9H, m, $\left.3 \mathrm{CH}_{2} \mathrm{CH}_{3}\right), 2.67$ $\left(4 \mathrm{H}, \mathrm{q}, J=7.2 \mathrm{~Hz}, 2 \underline{\mathrm{CH}}_{2} \mathrm{CH}_{3}\right), 4.22\left(2 \mathrm{H}, \mathrm{q}, J=7.2 \mathrm{~Hz}, \underline{\mathrm{CH}}_{2} \mathrm{CH}_{3}\right), 4.54\left(2 \mathrm{H}, \mathrm{s}, \mathrm{CH}_{2}-\mathrm{N}\right), 7.09$

Bull. Chem. Soc. Ethiop. 2019, 33(1) 
$(2 \mathrm{H}, \mathrm{d}, J=8.4 \mathrm{~Hz}, \mathrm{ArH}), 7.16(2 \mathrm{H}, \mathrm{d}, J=8.4 \mathrm{~Hz}, \operatorname{ArH}), 7.24(2 \mathrm{H}, \mathrm{d}, J=8.4 \mathrm{~Hz}, \operatorname{ArH}), 7.71(2 \mathrm{H}$, $\mathrm{d}, J=8.8 \mathrm{~Hz}, \mathrm{ArH}), 8.01(1 \mathrm{H}, \mathrm{s}, \mathrm{NH})$.

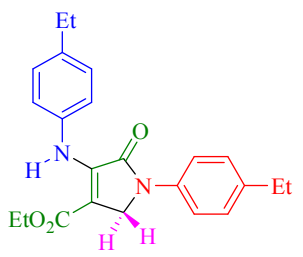

Ethyl 1-(4-bromophenyl)-3-(butylamino)-2,5-dihydro-2-oxo-1H-pyrrole-4-carboxylate (5q). Yield: 78\%; m.p. 93-95 ${ }^{\circ} \mathrm{C}$; ${ }^{1} \mathrm{H}$ NMR (400 MHz, $\left.\mathrm{CDCl}_{3}\right): 0.97\left(3 \mathrm{H}, \mathrm{t}, J=7.2 \mathrm{~Hz}, \mathrm{CH}_{3}\right), 1.35$ $\left(3 \mathrm{H}, \mathrm{t}, J=7.2 \mathrm{~Hz}, \mathrm{OCH}_{2} \mathrm{CH}_{3}\right), 1.43\left(2 \mathrm{H}\right.$, sextet, $\left.J=7.6 \mathrm{~Hz}, \mathrm{CH}_{2}\right), 1.61(2 \mathrm{H}$, quintet, $J=7.6 \mathrm{~Hz}$, $\left.\mathrm{CH}_{2}\right), 3.87\left(2 \mathrm{H}, \mathrm{t}, J=7.2 \mathrm{~Hz}, \underline{\mathrm{CH}_{2}}-\mathrm{NH}\right), 4.28\left(2 \mathrm{H}, \mathrm{q}, J=7.2 \mathrm{~Hz}, \mathrm{OCH}_{2} \mathrm{CH}_{3}\right), 4.40\left(2 \mathrm{H}, \mathrm{s}, \underline{\mathrm{CH}}_{2}-\right.$ $\mathrm{N}), 6.72(1 \mathrm{H}$, br s, NH), $7.52(2 \mathrm{H}, \mathrm{d}, J=8.8 \mathrm{~Hz}, \mathrm{ArH}), 7.70(2 \mathrm{H}, \mathrm{d}, J=8.8 \mathrm{~Hz}, \mathrm{ArH})$.

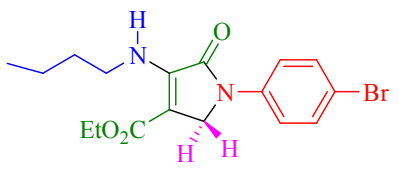

\section{RESULTS AND DISCUSSION}

To generality of this transformation, we investigated caffeine catalyzed four-component reaction between aniline ( $2 \mathrm{mmol})$, dimethyl acetylenedicarboxylate (DMAD) (1 mmol) and formaldehyde $(1.5 \mathrm{mmol})$ as a model reaction under mild conditions for the synthesis of dihydro-2-oxypyrroles. The amount of catalyst was studied with this method and in the absence of caffeine: a trace amount of this product was generated after $14 \mathrm{~h}$ (Table 1, entry 1). Good yields were obtained in the presence of a catalyst. The best amount of catalyst was $15 \mathrm{~mol} \%$ (Table 1, entry 4). The higher amount of catalyst did not increase the yields products (Table 1, entry 11) and the results are summarized in Table 1 . The effect of various solvents was investigated for this protocol $\mathrm{H}_{2} \mathrm{O}, \mathrm{CH}_{2} \mathrm{Cl}_{2}, \mathrm{CHCl}_{3}, \mathrm{EtOH}, \mathrm{MeOH}$ and $\mathrm{CH}_{3} \mathrm{CN}$. Herein, the reaction occurred efficiently to afford the corresponding polysubstituted dihydro-2-oxypyrroles

Table 1. Optimization of the reaction condition in the presence of different amounts of caffeine ${ }^{a}$.

\begin{tabular}{|r|c|c|c|c|}
\hline Entry & Caffeine (mol \%) & Solvent & Time (h) & Isolated yields (\%) \\
\hline 1 & Catalyst free & $\mathrm{MeOH}$ & 14 & Trace \\
\hline 2 & 5 & $\mathrm{MeOH}$ & 6 & 45 \\
\hline 3 & 10 & $\mathrm{MeOH}$ & 4 & $\mathbf{8 3}$ \\
\hline $\mathbf{4}$ & $\mathbf{1 5}$ & $\mathbf{M e O H}$ & $\mathbf{3}$ & 68 \\
\hline 5 & 15 & $\mathrm{EtOH}$ & 4 & 14 \\
\hline 6 & 15 & $\mathrm{H}_{2} \mathrm{O}$ & 8 & 40 \\
\hline 7 & 15 & $\mathrm{Solvent}$ free & 6 & 31 \\
\hline 8 & 15 & $\mathrm{CH}_{2} \mathrm{Cl}_{2}$ & 7 & 27 \\
\hline 9 & 15 & $\mathrm{CHCl}_{3}$ & 7 & 35 \\
\hline 10 & 15 & $\mathrm{CH}_{3} \mathrm{CN}$ & 6 & 89 \\
\hline 11 & 20 & $\mathrm{MeOH}$ & 3 & 77 \\
\hline 12 & 20 & EtOH & 4 & $\mathbf{8}$ \\
\hline
\end{tabular}

${ }^{a}$ Reaction conditions: aniline $(2 \mathrm{mmol})$, dimethyl acetylenedicarboxylate $(1 \mathrm{mmol})$ and formaldehyde $(1.5 \mathrm{mmol})$ and caffeine in various solvents at room temperature. 
in $88 \%$ yield when $15 \mathrm{~mol} \%$ caffeine was used in $\mathrm{MeOH}$ at room temperature (Table 1, entry 4). The efficiency of caffeine was demonstrated by synthesizing polysubstituted dihydro-2oxypyrroles via four-component condensation using a series aromatic/aliphatic amines (1 and $\mathbf{3}$, $2 \mathrm{mmol})$, dialkyl acetylenedicarboxylate $(2,1 \mathrm{mmol})$ and formaldehyde $(4,1.5 \mathrm{mmol})$ at ambient temperature which results are shown in Table 2.

Table 2. Caffeine catalyzed synthesis of polysubstituted dihydro-2-oxypyrroles.

\begin{tabular}{|c|c|c|c|c|c|c|c|c|}
\hline Entry & $\mathrm{R}^{1}$ & $\mathrm{R}^{2}$ & $\mathrm{Ar}$ & Product & Time (h) & Yield $(\%)^{a}$ & M.p. ${ }^{\circ} \mathrm{C}$ & Lit. M.p. C \\
\hline 1 & $\mathrm{Ph}$ & $\mathrm{Me}$ & $\mathrm{Ph}$ & $5 a$ & 3 & 88 & $154-156$ & $155-156[11]$ \\
\hline 2 & $\mathrm{Ph}$ & Et & $\mathrm{Ph}$ & $5 \mathbf{b}$ & 3 & 86 & $140-141$ & $138-140[15]$ \\
\hline 3 & 4-F- $\mathrm{C}_{6} \mathrm{H}_{4}$ & $\mathrm{Me}$ & 4-F- $\mathrm{C}_{6} \mathrm{H}_{4}$ & $5 c$ & 2.5 & 87 & 164-165 & $163-165$ [12] \\
\hline 4 & $4-\mathrm{F}-\mathrm{C}_{6} \mathrm{H}_{4}$ & Et & $4-\mathrm{F}-\mathrm{C}_{6} \mathrm{H}_{4}$ & 5d & 2.5 & 88 & $173-174$ & $172-174$ [13] \\
\hline 5 & 4-Br- $\mathrm{C}_{6} \mathrm{H}_{4}$ & $\mathrm{Me}$ & $4-\mathrm{Br}-\mathrm{C}_{6} \mathrm{H}_{4}$ & $5 e$ & 4 & 80 & $177-180$ & $175-177$ [13] \\
\hline 6 & 4- $\mathrm{Br}-\mathrm{C}_{6} \mathrm{H}_{4}$ & $\mathrm{Et}$ & 4-Br- $\mathrm{C}_{6} \mathrm{H}_{4}$ & $5 f$ & 5 & 78 & $168-170$ & $169-171[15]$ \\
\hline 7 & $4-\mathrm{OMe}-\mathrm{C}_{6} \mathrm{H}_{4}$ & $\mathrm{Me}$ & $4-\mathrm{OMe}-\mathrm{C}_{6} \mathrm{H}_{4}$ & $5 \mathrm{~g}$ & 3.5 & 85 & $175-177$ & $172-175[13]$ \\
\hline 8 & $4-\mathrm{OMe}-\mathrm{C}_{6} \mathrm{H}_{4}$ & Et & $4-\mathrm{OMe}-\mathrm{C}_{6} \mathrm{H}_{4}$ & $5 \mathrm{~h}$ & 4.5 & 87 & $150-152$ & $152-154[14]$ \\
\hline 9 & $4-\mathrm{Cl}-\mathrm{C}_{6} \mathrm{H}_{4}$ & $\mathrm{Me}$ & $4-\mathrm{Cl}-\mathrm{C}_{6} \mathrm{H}_{4}$ & $5 i$ & 4 & 83 & $170-172$ & $171-173$ [13] \\
\hline 10 & $4-\mathrm{Cl}-\mathrm{C}_{6} \mathrm{H}_{4}$ & $\mathrm{Et}$ & $4-\mathrm{Cl}-\mathrm{C}_{6} \mathrm{H}_{4}$ & $5 \mathbf{j}$ & 4.5 & 85 & 166-168 & $168-170[13]$ \\
\hline 11 & 4-Me- $\mathrm{C}_{6} \mathrm{H}_{4}$ & $\mathrm{Me}$ & $4-\mathrm{Me}-\mathrm{C}_{6} \mathrm{H}_{4}$ & $5 k$ & 3 & 89 & $176-178$ & $177-178$ [14] \\
\hline 12 & 4-Me- $\mathrm{C}_{6} \mathrm{H}_{4}$ & Et & $4-\mathrm{Me}-\mathrm{C}_{6} \mathrm{H}_{4}$ & 51 & 3 & 86 & $132-134$ & $131-132$ [15] \\
\hline 13 & 4-Et- $\mathrm{C}_{6} \mathrm{H}_{4}$ & $\mathrm{Me}$ & 4-Et- $\mathrm{C}_{6} \mathrm{H}_{4}$ & $5 \mathrm{~m}$ & 3.5 & 82 & $126-128$ & $124-125$ [19] \\
\hline 14 & 4-Et- $\mathrm{C}_{6} \mathrm{H}_{4}$ & $\mathrm{Et}$ & 4-Et- $\mathrm{C}_{6} \mathrm{H}_{4}$ & $5 n$ & 3.5 & 84 & 102-104 & $102-104$ [19] \\
\hline 15 & $\mathrm{n}-\mathrm{C}_{4} \mathrm{H}_{9}$ & $\mathrm{Me}$ & $\mathrm{Ph}$ & 50 & 4 & 86 & $60-62$ & $60[11]$ \\
\hline 16 & $\mathrm{n}-\mathrm{C}_{4} \mathrm{H}_{9}$ & $\mathrm{Me}$ & $3,4-\mathrm{Cl}_{2}-\mathrm{C}_{6} \mathrm{H}_{3}$ & $5 p$ & 4.5 & 80 & $96-98$ & $97-99$ [14] \\
\hline 17 & $\mathrm{n}-\mathrm{C}_{4} \mathrm{H}_{9}$ & Et & $4-\mathrm{Br}-\mathrm{C}_{6} \mathrm{H}_{4}$ & $5 q$ & 5 & 78 & $93-95$ & $94-96$ [14] \\
\hline 18 & $\mathrm{PhCH}_{2}$ & $\mathrm{Me}$ & $\mathrm{Ph}$ & $5 r$ & 3.5 & 84 & $140-142$ & $140-141$ [15] \\
\hline 19 & $\mathrm{PhCH}_{2}$ & $\mathrm{Me}$ & $4-\mathrm{F}-\mathrm{C}_{6} \mathrm{H}_{4}$ & $5 s$ & 3.5 & 85 & 166-167 & $166-168$ [14] \\
\hline 20 & $\mathrm{PhCH}_{2}$ & Et & $\mathrm{Ph}$ & $5 t$ & 4 & 82 & $130-132$ & $130-132[15]$ \\
\hline
\end{tabular}

${ }^{a}$ Isolated yield.

Table 3. Comparison of catalytic ability some of catalysts reported in the literature for synthesis of polysubstituted dihydro-2-oxypyrroles ${ }^{a}$.

\begin{tabular}{|c|c|c|c|c|c|}
\hline Entry & Product & Catalyst & Conditions & Time/Yield (\%) & Reference \\
\hline 1 & $5 a$ & $\mathrm{I}_{2}$ & $\mathrm{MeOH}$, r.t. & $1 \mathrm{~h} / 82$ & [11] \\
\hline 2 & $5 a$ & $\mathrm{InCl}_{3}$ & $\mathrm{MeOH}$, r.t. & $3 \mathrm{~h} / 85$ & [12] \\
\hline 3 & $5 a$ & {$\left[\mathrm{n}-\mathrm{Bu}_{4} \mathrm{~N}\right]\left[\mathrm{HSO}_{4}\right]$} & $\mathrm{MeOH}$, r.t. & $4 \mathrm{~h} / 88$ & [13] \\
\hline 4 & $5 \mathrm{a}$ & $\mathrm{Al}\left(\mathrm{H}_{2} \mathrm{PO}_{4}\right)_{3}$ & $\mathrm{MeOH}$, r.t. & $5 \mathrm{~h} / 81$ & [14] \\
\hline 5 & $5 a$ & $\mathrm{Cu}(\mathrm{OAc})_{2} \cdot \mathrm{H}_{2} \mathrm{O}$ & $\mathrm{MeOH}$, r.t. & $6 \mathrm{~h} / 91$ & [16] \\
\hline 6 & $5 a$ & $\mathrm{ZrCl}_{4}$ & $\mathrm{MeOH}$, r.t. & $4 \mathrm{~h} / 84$ & {$[18]$} \\
\hline 7 & $5 a$ & Caffeine & $\mathrm{MeOH}$, r.t. & $3 \mathrm{~h} / 88$ & This work \\
\hline 8 & $5 \mathrm{~h}$ & $\mathrm{I}_{2}$ & $\mathrm{MeOH}$, r.t. & $1 \mathrm{~h} / 81$ & {$[11]$} \\
\hline 9 & $5 \mathrm{~h}$ & $\mathrm{InCl}_{3}$ & $\mathrm{MeOH}$, r.t. & $3 \mathrm{~h} / 85$ & [12] \\
\hline 10 & $5 \mathrm{~h}$ & {$\left[\mathrm{n}-\mathrm{Bu}_{4} \mathrm{~N}\right]\left[\mathrm{HSO}_{4}\right]$} & $\mathrm{MeOH}$, r.t. & $4 \mathrm{~h} / 86$ & [13] \\
\hline 11 & $5 \mathrm{~h}$ & $\mathrm{Al}\left(\mathrm{H}_{2} \mathrm{PO}_{4}\right)_{3}$ & $\mathrm{MeOH}$, r.t. & $5 \mathrm{~h} / 80$ & [14] \\
\hline 12 & $5 \mathrm{~h}$ & $\mathrm{Cu}(\mathrm{OAc})_{2} \cdot \mathrm{H}_{2} \mathrm{O}$ & $\mathrm{MeOH}$, r.t. & $5 \mathrm{~h} / 85$ & [16] \\
\hline 13 & $5 \mathrm{~h}$ & $\mathrm{ZrCl}_{4}$ & $\mathrm{MeOH}$, r.t. & $3.5 \mathrm{~h} / 83$ & [18] \\
\hline 14 & $5 \mathrm{~h}$ & Caffeine & $\mathrm{MeOH}$, r.t. & $3 \mathrm{~h} / 86$ & This work \\
\hline
\end{tabular}

${ }^{a}$ Based on the four-component reaction of aniline, dimethylacetylenedicarboxylate, formaldehyde.

Proposed mechanism for the synthesis of polysubstituted dihydro-2-oxypyrroles is illustrated in Scheme 2. Initially, the amine 3 reacts with formaldehyde 4 in the presence of 
caffeine to form imine A. Also, the Michael reaction between amine $\mathbf{1}$ and dialkylacetylenedicarboxylate $\mathbf{2}$ gives enamine B. Activated imine A undergoes a Mannich type reaction with enamine $\mathbf{B}$ to generate intermediate $\mathbf{C}$, which converts to more stable tautomeric form $\mathbf{D}$. The intramolecular cyclization in intermediate $\mathbf{D}$ that in the final step, it tautomerizes into the corresponding polysubstituted dihydro-2-oxypyrroles $\mathbf{5}$.

Comparison of catalytic ability some of catalysts reported in the literature for synthesis of polysubstituted dihydro-2-oxypyrroles are shown in Table 3. This study reveals that caffeine has shown its extraordinary potential to be an alternative efficient, green, biodegradable and an inexpensive catalyst for the one-pot clean synthesis of these biologically active heterocyclic compounds, in addition to good to high yields is the notable advantages this present methodology.

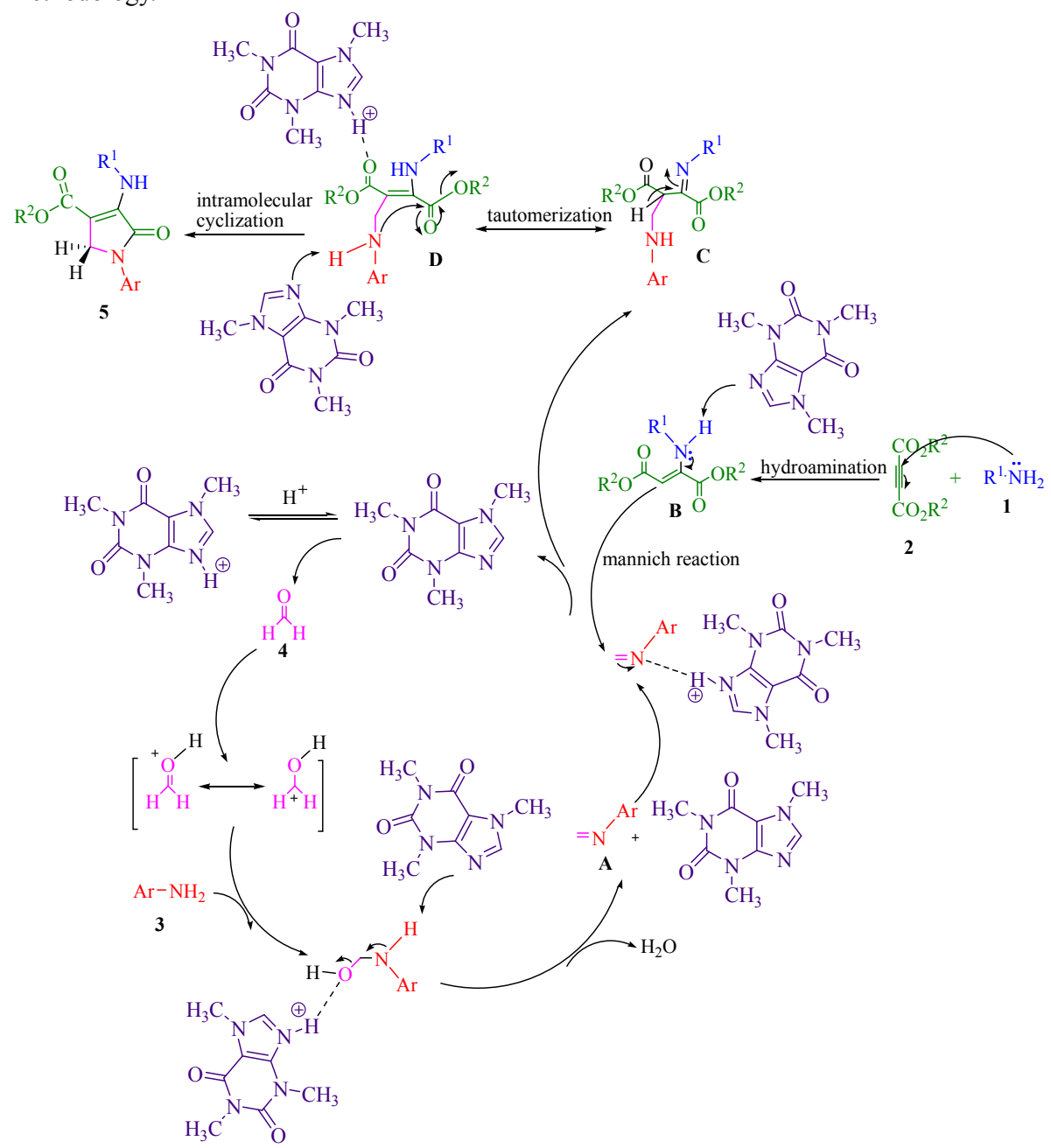

Scheme 2. Proposed mechanistic route for the synthesis of polysubstituted dihydro-2oxypyrroles. 


\section{CONCLUSION}

In summary, a mild, convenient and efficient procedure for the one-pot synthesis of polysubstituted dihydro-2-oxypyrroles by using of a catalytic amount of caffeine as a green catalyst under ambient temperature is reported. The use of a natural, biodegradable and inexpensive catalyst, along with simple work up, short reaction times and good to high yields, provides a compelling method to prepare these biologically active compounds.

\section{ACKNOWLEDGEMENT}

We gratefully acknowledge financial support from Young Researchers and Elite Club, Shiraz Branch, Islamic Azad University of Shiraz.

\section{REFERENCES}

1. Jones, R.A.; Bean, G.P. The Chemistry of Pyrroles, 1st ed., Academic Press: London; 1977.

2. Gossauer, A. In Methoden der Organichen Chemie (Houben-Weyl), 4th ed., Hetarene I, Teil 1 Pyrrole, Georg Thieme Verlag: New York; 1994; p 556-798.

3. Khajuria, R.; Dham, S.; Kapoor, K.K. Active methylenes in the synthesis of pyrrole motif: An imperative structural unit of Pharmaceuticals, Natural Products and Optoelectronic materials. RSC Adv. 2016, 6, 37039-37066.

4. Borthwick, A.D.; zzj.Crame, A.; Ertl, P.F.; Exall, A.M.; Haley, T.M.; Hart, G.J.; Mason, A.M.; Pennell, A.M.K.; Singh, O.M.P.; Weingarten, G.G.; Woolven, J. Design and synthesis of pyrrolidine-5,5-trans-lactams (5-oxohexahydropyrrolo[3,2-b]pyrroles) as novel mechanism-based inhibitors of human cytomegalovirus protease. 2 . Potency and chirality. $J$. Med. Chem. 2002, 45, 1-18.

5. Alp, C.; Ekinci, D.; Gultekin, M.S.; Senturk, M.; Sahin, E.; Kufrevioglu, O. A novel and one-pot synthesis of new 1-tosyl pyrrol-2-one derivatives and analysis of carbonic anhydrase inhibitory potencies. Bioorg. Med. Chem. 2010, 18, 4468-4474.

6. Shiraki, R.; Sumino, A.; Tadano, K.; Ogawa, S. Total synthesis of PI-091. Tetrahedron Lett. 1995, 36, 5551-5554.

7. Lampe, Y.L.; Chou, R.G.; Hanna, R.G.; DiMeo, S.V.; Erhardt, P.W.; Hagedorn, A.A.; Ingebretsen, W.R.; Cantor, E. (Imidazolylphenyl) pyrrol- 2-one inhibitors of cardiac cAMP phosphodiesterase. J. Med. Chem. 1993, 36, 1041-1047.

8. Chen, Y.; Zeng, D.X.; Xie, N.; Dang, Y.Z. Study on photochromism of diarylethenes with a 2, 5-dihydropyrrole bridging unit: a convenient preparation of 3, 4-diarylpyrroles from 3,4diaryl-2,5-dihydropyrroles. J. Org. Chem. 2005, 70, 5001-5005.

9. Singh, S.B.; Goetz, M.A.; Jones, E.T.; Billes, G.F.; Giacobbe, R.A.; Herranz, L.; StevensMiles S.; Williams D.L. a novel antagonist of endothelin receptor. J. Org. Chem. 1995, 60, 7040-7042.

10. Peifer, C.; Selig, R.; Kinkel, K.; Ott, D.; Totzke, F.; Scha chtele, C.; Heidenreich, R.; Ro cken, M.; Schollmeyer, D.; Laufer, S. Design, synthesis, and biological evaluation of novel 3-aryl-4-(1H-indole-3yl)-1,5-dihydro-2H-pyrrole-2-ones as vascular endothelial growth factor receptor (VEGF-R) inhibitors. J. Med. Chem. 2008, 51, 3814-3824.

11. Khan, A.T.; Ghosh, A.; Musawwer Khan, M. One-pot four-component domino reaction for the synthesis of substituted dihydro-2-oxypyrrole catalyzed by molecular iodine. Tetrahedron Lett. 2012, 53, 2622-2626.

12. Sajadikhah, S.S.; Maghsoodlou, M.T.; Hazeri, N. A simple and efficient approach to one-pot synthesis of mono- and bis-N-aryl-3-aminodihidropyrrol-2-one-4-carboxylate catalyzed by $\mathrm{InCl}_{3}$. Chin. Chem. Lett. 2014, 25, 58-60. 
13. Sajadikhah, S.S.; Hazeri, N. Coupling of amines, dialkyl acetylenedicarboxylaes and formaldehyde promoted by $\left[\mathrm{n}-\mathrm{Bu}_{4} \mathrm{~N}\right]\left[\mathrm{HSO}_{4}\right]$ : An efficient synthesis of highly functionalized dihydro-2-oxopyrrols and bis-dihydro-2-oxopyrroles. Res. Chem. Intermed. 2014, 40, 737748 .

14. Sajadikhah, S.S.; Hazeri, N.; Maghsoodlou, M.T.; Habibi-Khorassani, S.M.; Beigbabaei, A.; Willis, A.C. $\mathrm{Al}\left(\mathrm{H}_{2} \mathrm{PO}_{4}\right)_{3}$ as an efficient and reusable catalyst for the multi-component synthesis of highly functionalized piperidines and dihydro-2-oxypyrroles. 2013, J. Iran. Chem. Soc. 10, 863-871.

15. Zhu, Q.; Jiang, H.; Li, J.; Liu, S.; Xia, C.; Zhang, M. Concise and versatile multicomponent synthesis of multisubstituted polyfunctional dihydropyrroles. J. Comb. Chem. 2009, 11, 685696.

16. Lv, L.; Zheng, S.; Cai, X.; Chen, Z.; Zhu, Q.; Liu, S. Development of four-component synthesis of tetra- and pentasubstituted polyfunctional dihydropyrroles: Free permutation and combination of aromatic and aliphatic amines. J. ACS Comb. Sci. 2013, 15, 183-192.

17. Sajadikhah, S.S.; Hazeri, N.; Maghsoodlou, M.T. A one-pot multi-component synthesis of $\mathrm{N}$-aryl-3-aminodihydropyrrol-2-one-4-carboxylates catalysed by oxalic acid dihydrate. $J$. Chem. Res. 2013, 37, 40-42.

18. Sajadikhah, S.S.; Maghsoodlou, M.T.; Hazeri, N.; Mohamadian-Souri, S. $\mathrm{ZrCl}_{4}$ as an efficient catalyst for one-pot four-component synthesis of polysubstituted dihydropyrrol-2ones. Res. Chem. Intermed. 2016, 42, 2805-2814.

19. Salehi, N.; Mirjalili, B.B.F. Synthesis of highly substituted dihydro-2-oxopyrroles using $\mathrm{Fe}_{3} \mathrm{O}_{4} @$ nano-cellulose- $\mathrm{OPO}_{3} \mathrm{H}$ as a novel bio-based magnetic nanocatalyst. $R S C A d v$. 2017, 7, 30303-30309.

20. Nehlig, A.; Daval, J.L.; Debry, G. Caffeine and the central nervous system: mechanisms of action, biochemical, metabolic and psychostimulant effects. Brain Res. Rev. 1992, 17, 139170.

21. Bahrami, H.; Tabrizchi, M.; Farrokhpour, H. Protonation of caffeine: A theoretical and experimental study. Chem. Phys. 2013, 415, 222-227.

22. Mohamadpour, F. Green and convenient one-pot access to polyfunctionalized piperidine scaffolds via glutamic acid catalyzed Knoevenagel-intramolecular [4+2] aza-Diels-Alder imin-based multi-component reaction under ambient temperature. Polycycl. Aromat. Comp. 2018. DOI: $10.1080 / 10406638.2018 .1472111$.

23. Mohamadpour, F.; Maghsoodlou, M.T.; Heydari, R.; Lashkari, M. Copper(II) acetate monohydrate: An efficient and eco-friendly catalyst for the one-pot multi-component synthesis of biologically active spiropyrans and 1H-pyrazolo[1,2-b]phthalazine-5,10-dione derivatives under solvent-free conditions. Res. Chem. Intermed. 2016, 42, 7841-7853.

24. Zare Fekri, L.; Nikpassand, M.; Pourmirzajani, S.; Aghazadeh, B. Synthesis and characterization of amino glucose-functionalized silica-coated $\mathrm{NiFe}_{2} \mathrm{O}_{4}$ nanoparticles: A heterogeneous, new and magnetically separable catalyst for the solvent-free synthesis of pyrano[3,2-c]chromen-5(4H)-ones. RSC Adv. 2018, 8, 22313-22320.

25. Lashkari, M.; Heydari, R.; Mohamadpour, F. A facile approach for one-pot synthesis of $1 \mathrm{H}-$ pyrazolo[1,2-b]phthalazine-5,10-dione derivatives catalyzed by $\mathrm{ZrCl}_{4}$ as an efficient catalyst under solvent-free conditions. Iran. J. Sci. Technol. Trans. Sci. 2018, 42, 1191-1197.

26. Maghsoodlou, M.T.; Heydari, R.; Mohamadpour, F.; Lashkari, M. $\mathrm{Fe}_{2} \mathrm{O}_{3}$ as an environmentally benign natural catalyst for one-pot and solvent-free synthesis of spiro- $4 \mathrm{H}-$ pyran derivatives. Iran. J. Chem. Chem. Eng. 2017, 36, 31-38.

27. Nikpassand, M.; Zare Fekri, L. One-pot synthesis of 9-arylxanthenediones and 9pyrazoloxanthenediones using [DBU] OAc, Russ. J. Gen. Chem. 2017, 87, 816-820.

28. Zahedi, N.; Javid, A.; Mohammadi, M.K.; Tavakkoli, H. Microwave-promoted solvent free one-pot synthesis of triazolo[1,2-a] indazole-triones catalyzed by silica-supported $\mathrm{La} 0.5 \mathrm{Ca}$ 
$0.5 \mathrm{CrO}_{3}$ nanoparticles as a new and reausable provskitetype oxide. Bull. Chem. Soc. Ethiop. 2018, 32, 239-248.

29. Zare Fekri, L.; Nikpassand, M.; Movaghari, M. $\mathrm{Fe}^{+3}$-montmorillonite K10: As an effective and reusable catalyst for the synthesis of 3, 4-dihydropyrimidin-2 $(1 H)$-ones and-thiones. Bull. Chem. Soc. Ethiop. 2017, 31, 313-321.

30. Zekavati, R.; Doulah, A.; Mohammadi, M.K.; Shirali, S. Solvent free synthesis of Nalkylated imino indoline-2-ones derivatives under microwave irradiation. Bull. Chem. Soc. Ethiop. 2018, 32, 393-398.

31. Bahrami, F.; Nikpassand, M. Potassium 2-oxoimidazolidine-1, 3-diide: An effective and new catalyst for the grinding synthesis of $(1 H$-indol-3-yl $)$ methyl-2H-indan-1,3-diones. Bull. Chem. Soc. Ethiop. 2018, 32, 399-405.

32. Maghsoodlou, M.T.; Heydari, R.; Lashkari, M.; Mohamadpour, F. Clean and one-pot synthesis of 3, 4-dihydropyrimidin-2-( $1 H)$-ones/tiones derivatives using maleic acid as an efficient and environmentally benign nature di-functional Brønsted acid catalyst under solvent-free conditions. Indian. J. Chem. 56B 2017, 56B, 160-164.

33. Mohamadpour, F.; Lashkari, M. Three-component reaction of $\beta$-keto esters, aromatic aldehydes and urea/thiourea promoted by caffeine: A green and natural, biodegradable catalyst for eco-safe Biginelli synthesis of 3,4-dihydropyrimidin-2-(1H)-ones/tiones derivatives under solvent-free conditions. J. Serb. Chem. Soc. 2018, 83, 673-684. 